\title{
Precision validation of MIPAS-Envisat products
}

\author{
C. Piccolo and A. Dudhia \\ Atmospheric, Oceanic and Planetary Physics, University of Oxford, Oxford, UK \\ Received: 21 December 2006 - Published in Atmos. Chem. Phys. Discuss.: 18 January 2007 \\ Revised: 5 April 2007 - Accepted: 5 April 2007 - Published: 17 April 2007
}

\begin{abstract}
This paper discusses the variation and validation of the precision, or estimated random error, associated with the ESA Level 2 products from the Michelson Interferometer for Passive Atmospheric Sounding (MIPAS).

This quantity represents the propagation of the radiometric noise from the spectra through the retrieval process into the Level 2 profile values. The noise itself varies with time, steadily rising between ice decontamination events, but the Level 2 precision has a greater variation due to the atmospheric temperature which controls the total radiance received. Hence, for all species, the precision varies latitudinally/seasonally with temperature, with a small superimposed temporal structure determined by the degree of ice contamination on the detectors.

The precision validation involves comparing two MIPAS retrievals at the intersections of ascending/descending orbits. For 5 days per month of full resolution MIPAS operation, the standard deviation of the matching profile pairs is computed and compared with the precision given in the MIPAS Level 2 data, except for $\mathrm{NO}_{2}$ since it has a large diurnal variation between ascending/descending intersections. Even taking into account the propagation of the pressure-temperature retrieval errors into the VMR retrieval, the standard deviation of the matching pairs is usually a factor 1-2 larger than the precision. This is thought to be due to effects such as horizontal inhomogeneity of the atmosphere and instability of the retrieval.
\end{abstract}

\section{Introduction}

The Michelson Interferometer for Passive Atmospheric Sounding (MIPAS) (Fischer et al., 2007 ${ }^{1}$ ) is an infrared limb-sounding Fourier transform interferometer on board the Envisat satellite, launched in March 2002 (ESA, 2000). It acquires spectra over the range $685-2410 \mathrm{~cm}^{-1}(14.5-$ $4.1 \mu \mathrm{m}$ ), which includes the vibration-rotation bands of many molecules of interest. It is capable of measuring continuously around an orbit from pole to pole in both day and night and nearly complete global coverage is obtained in $24 \mathrm{~h}$.

From July 2002 until March 2004 MIPAS was operated at full spectral resolution (sampled at $0.025 \mathrm{~cm}^{-1}$ ) with a nominal limb-scanning sequence of 17 steps from $68-6 \mathrm{~km}$ with $3 \mathrm{~km}$ tangent height spacing in the troposphere and stratosphere, generating complete scans spaced approximately every $500 \mathrm{~km}$ along the orbit. Using the 17 spectra from each scan as input, the ESA Level 2 (L2) processing uses a global least-squares fit algorithm (Ridolfi et al., 2000; Raspollini et al., 2006) to retrieve profiles of atmospheric temperature and pressure (pT) followed sequentially by the concentrations of 6 "key species": $\mathrm{H}_{2} \mathrm{O}, \mathrm{O}_{3}, \mathrm{HNO}_{3}, \mathrm{CH}_{4}, \mathrm{~N}_{2} \mathrm{O}$ and $\mathrm{NO}_{2}$. These are the $\mathrm{L} 2$ products, which have now been generated for the entire full resolution dataset.

MIPAS operations were suspended in March 2004 following problems with the interferometer slide mechanism. Operations resumed in January 2005 with a reduced spectral sampling (about $0.061 \mathrm{~cm}^{-1}$ for $8.2 \mathrm{~cm}$ maximum optical path difference of the interferometer), a reduced duty cycle and a different limb scanning sequence, but only data from the full resolution mission are discussed here.

\footnotetext{
${ }^{1}$ Fischer, H., Birk, M., Blom, C., et al.: MIPAS: an instrument for atmospheric and climate research, Atmos. Chem. Phys. Discuss., in preparation, 2007.
} 

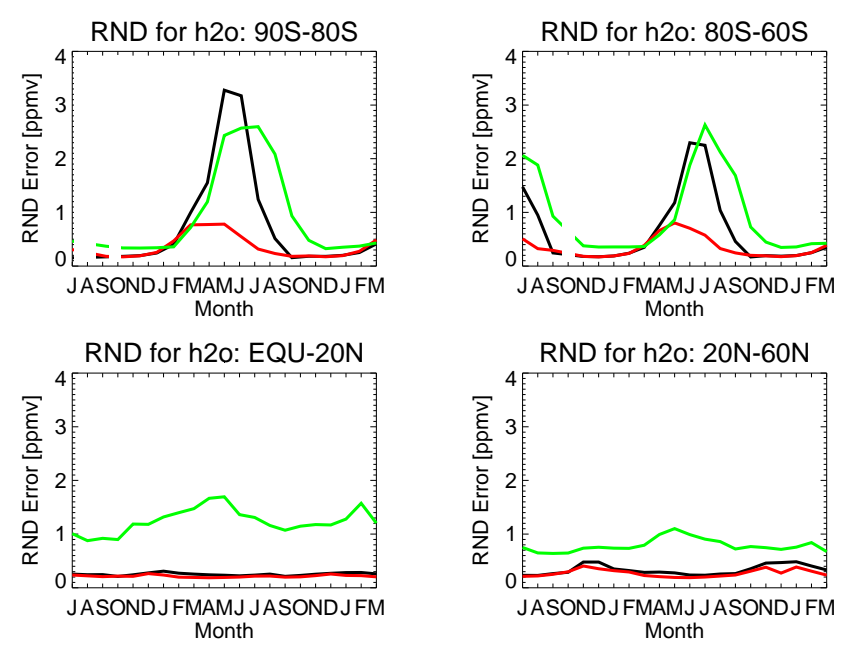
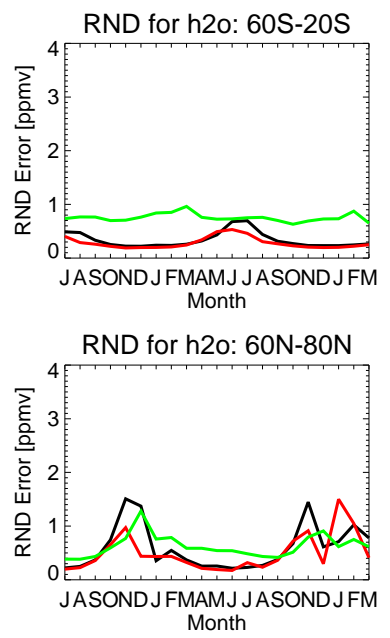
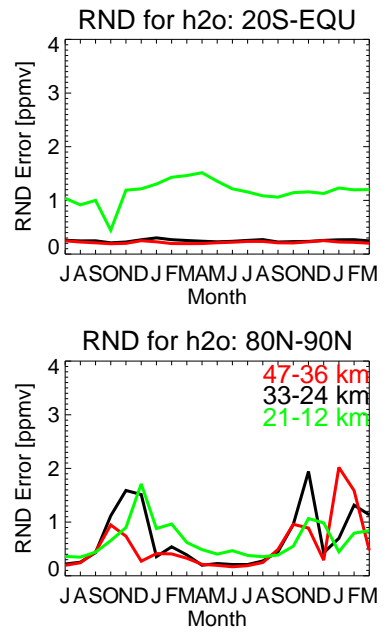

Fig. 1. Time series of the precision derived from MIPAS L2 data for $\mathrm{H}_{2} \mathrm{O}$ [ppmv] from July 2002 to March 2004, split in eight latitude bands. The red line shows the precision averaged between $47 \mathrm{~km}$ and $36 \mathrm{~km}$ tangent heights, the black line between $33 \mathrm{~km}$ and $24 \mathrm{~km}$ and the green line between $21 \mathrm{~km}$ and $12 \mathrm{~km}$. The gaps in the southern hemisphere in July-August 2002 and October 2002 are caused by missing Antarctic L2 profiles.

This paper presents the validation of the precision values associated with the ESA L2 MIPAS data. Section 2 discusses the sources of the random error in the retrieved profiles, and the dependence upon the radiometric noise and atmospheric temperature; Sect. 3 describes the method used for validating the precision values; Sect. 4 presents the results of the validation and Sect. 5 the conclusions drawn from this study.

\section{Precision}

Each L2 profile $\boldsymbol{x}$ has an associated covariance matrix $\mathbf{V}_{x}$ representing the mapping of the radiometric noise through the retrieval

$\mathbf{V}_{x}=\left(\mathbf{K}^{T} \mathbf{V}_{n}^{-1} \mathbf{K}\right)^{-1}$

where $\mathbf{V}_{n}$ is the covariance matrix of the noise and $\mathbf{K}$ is the jacobian matrix (Raspollini et al., 2006). The square roots of the diagonal elements of $\mathbf{V}_{x}$ represent the error in the corresponding elements of the profile $\boldsymbol{x}$ (units of Kelvin for temperature and parts per million by volume, ppmv, for volume mixing ratios (VMR)). These are the precision values to be validated. The off-diagonal elements $\left(\mathbf{V}_{x}\right)_{i j}$ of $\mathbf{V}_{x}$ provide the correlations between values $i$ and $j$ of the profile $\boldsymbol{x}$.

In the optically thin case if we assume that the radiance $R$ is proportional to the product of the Plank function $B(T)$, the absorption cross-section $k$, the air density $n$ and the volume mixing ratio $x$ :

$R=k n \times B(T) \propto k \times B(T) / T$, then the precision $\delta x$ (in ppmv) is proportional to the noise $\delta R$ and inversely proportional to the Planck function over temperature:

$\delta x \propto \frac{\delta R}{B(T) / T}$.

In the infrared the term $B(T) / T$ is dominated by Planck function $B(T)$. Therefore we use the absolute error rather than fractional error $\delta x / x$ since this removes the dependence on target species concentration.

\subsection{Precision variability}

The precision values given in the MIPAS L2 data have been averaged for every 5 days per month of full resolution MIPAS operation and are not expected to vary significantly over shorter timescales. Figures 1 and 2 show an example of the time series of the precision for $\mathrm{H}_{2} \mathrm{O}$ and $\mathrm{HNO}_{3}$, respectively (some unrealistic values have been filtered out).

The variability with time of the precision is evident for both species in the polar latitudes bands. All other species show a similar behaviour. There are distinct peaks in the polar winter cases for the three height ranges in both hemispheres, although in the second arctic winter there is a clear dip in the middle of the peak during December 2003. The mid latitude and equatorial regions do not show strong variations with time.

\subsection{Atmospheric temperature variability}

As explained previously, the precision value in the L2 products is expected to be inversely proportional to the Planck 

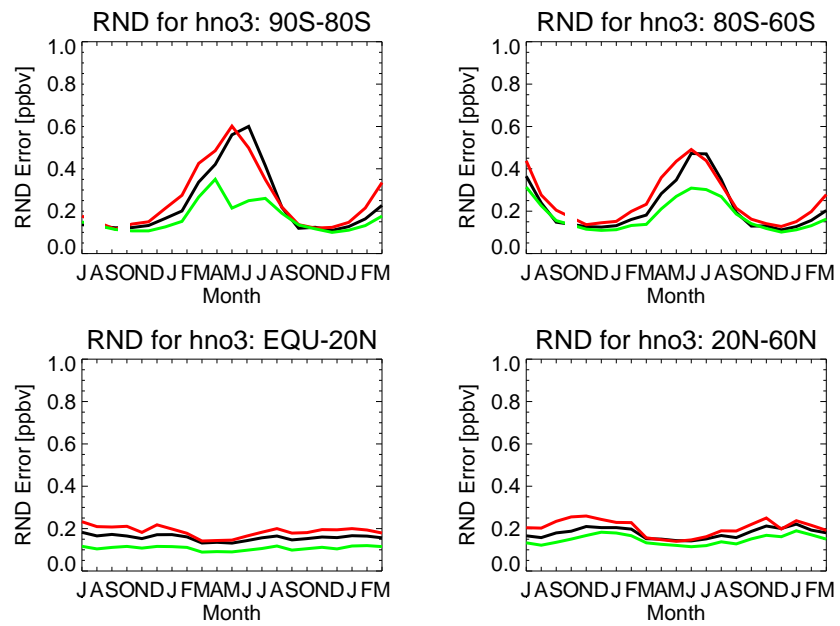

Fig. 2. As Fig. 1, except for $\mathrm{HNO}_{3}$ [ppbv].

function, i.e. smaller retrieval errors for warmer atmospheres. Figure 3 shows the time series of MIPAS-retrieved temperature.

Comparing Fig. 3 to Figs. 1 and 2 it is clear that the atmospheric temperature explains much of the variability in the precision of the MIPAS L2 products, including the decrease in random error observed in the arctic in December 2002 and 2003 which can be attributed to a stratospheric warming.

\subsection{Noise variability}

The other component of the precision value is the radiometric noise, expressed as a Noise Equivalent Spectral Radiance (NESR), which varies with radiometric gain (determined by the degree of ice contamination on the detectors, Kleinert et al., 2006) but is also slightly dependent on the atmospheric signal: larger radiance also implies larger NESR (ESA, 2000). Figure 4 shows the time series of the NESR derived from MIPAS L1B data (i.e. radiometrically and spectrally calibrated geolocated spectra) for polar latitudes at $68 \mathrm{~km}$ and $33 \mathrm{~km}$ tangent altitudes, averaged over each of the 5 different spectral bands.

The A band signal shows the clearest dependence on the degree of ice-contamination, rising steadily between decontamination events, while the noise in the other bands is more sensitive to atmospheric radiance. The maxima in bands $A B$, B, C and D for 80 N-90 N in December 2002 and 2003 can be attributed to the stratospheric warming observed in the arctic in December 2002 and 2003.

So the $\mathrm{L} 2$ precision value is influenced by variations in atmospheric temperature in two ways in opposing directions: increasing temperature results in an increased signal but also an increased noise. However, since the signal variation is nearer $\pm 100 \%$ while the seasonal noise variations are only
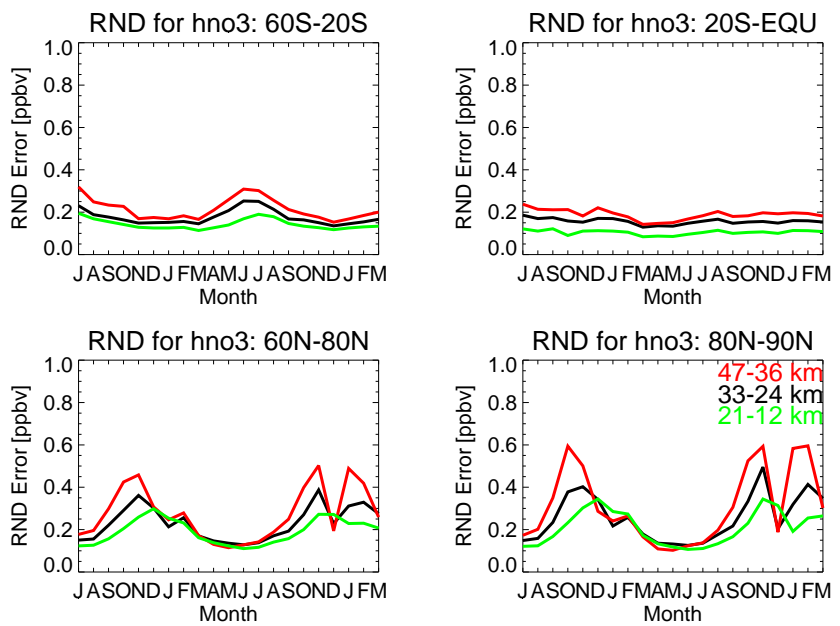

of the order of $\pm 20 \%$, the former effect dominates: higher temperatures result in more precise retrievals.

\section{Methodology of validation}

The precision of the retrievals may be defined as the dispersion of an ensemble of retrievals obtained from limb measurements of the same atmospheric state. Although, in practice, MIPAS does not make repeated measurements of the same limb path, an approximation is available from the pairs of measurements located at the intersections of the MIPAS viewing tracks from orbits a few hours apart. If the colocations are sufficiently close in space and time that atmospheric variations can be neglected, the actual precision of the retrievals can be estimated from the standard deviation of these pairs (Lambert et al., 1996).

Profile locations of ascending and descending tracks were matched to within $300 \mathrm{~km}$ in distance (compared to approximately $500 \mathrm{~km}$ distance between successive profiles along the orbit) for every 5 days per month of full resolution MIPAS operation. Limiting the comparisons of profile locations to $3 \mathrm{~h}$ time difference and $300 \mathrm{~km}$ horizontal difference produces regular matches at two latitudes, near the poles, $\sim 150$ matches in the $80 \mathrm{~S}-90 \mathrm{~S}$ and $\sim 200$ matches in the $80 \mathrm{~N}-$ $90 \mathrm{~N}$ regions. Extending the comparisons to $12 \mathrm{~h}$ time difference produces matches at three additional latitudes in the regions $20 \mathrm{~S}-60 \mathrm{~S}(\sim 150), 60 \mathrm{~S}-80 \mathrm{~S}(\sim 200)$ and $60 \mathrm{~N}-80 \mathrm{~N}$ $(\sim 350)$.

Figure 5 shows the locations of the MIPAS profiles for 30 July 2003.

For every tangent point in every pair of profiles, the difference $z=x_{1}-x_{2}$ was calculated, where $x_{1}$ and $x_{2}$ represent either temperature or VMR of the target species. However, to 

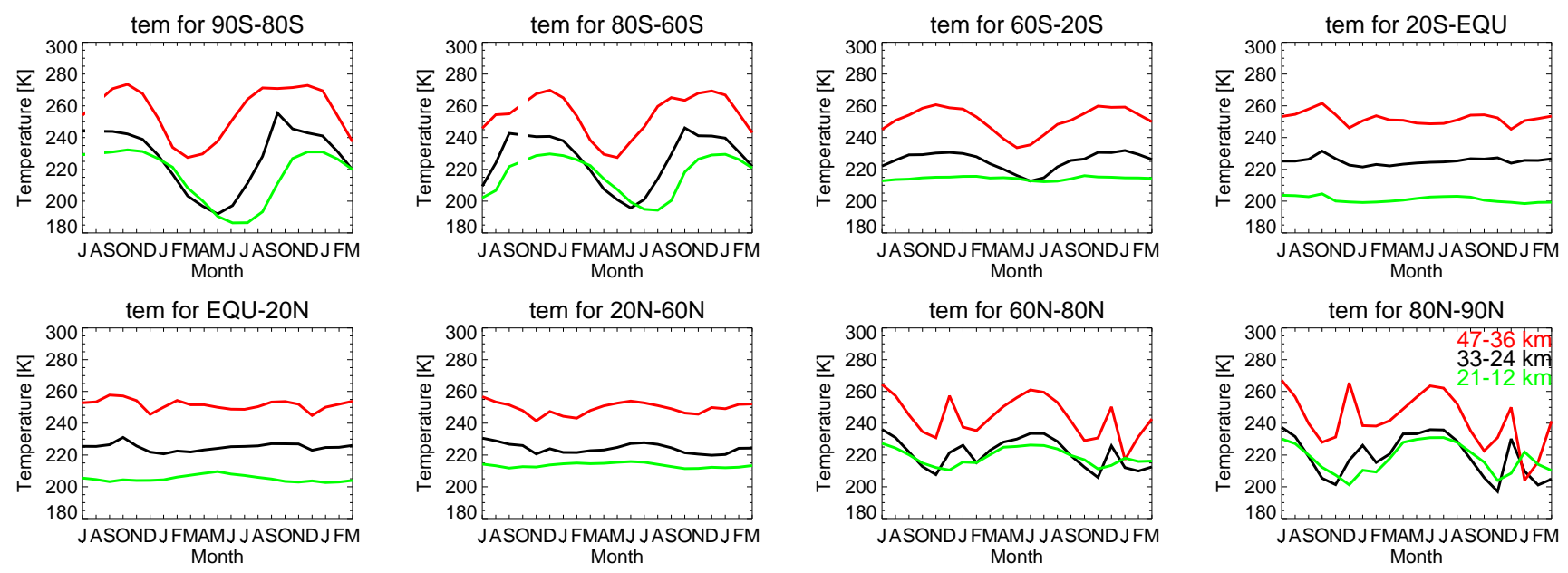

Fig. 3. Time series of retrieved temperature [K] derived from MIPAS L2 data, corresponding to precision variations shown in Figs. 1 and 2.
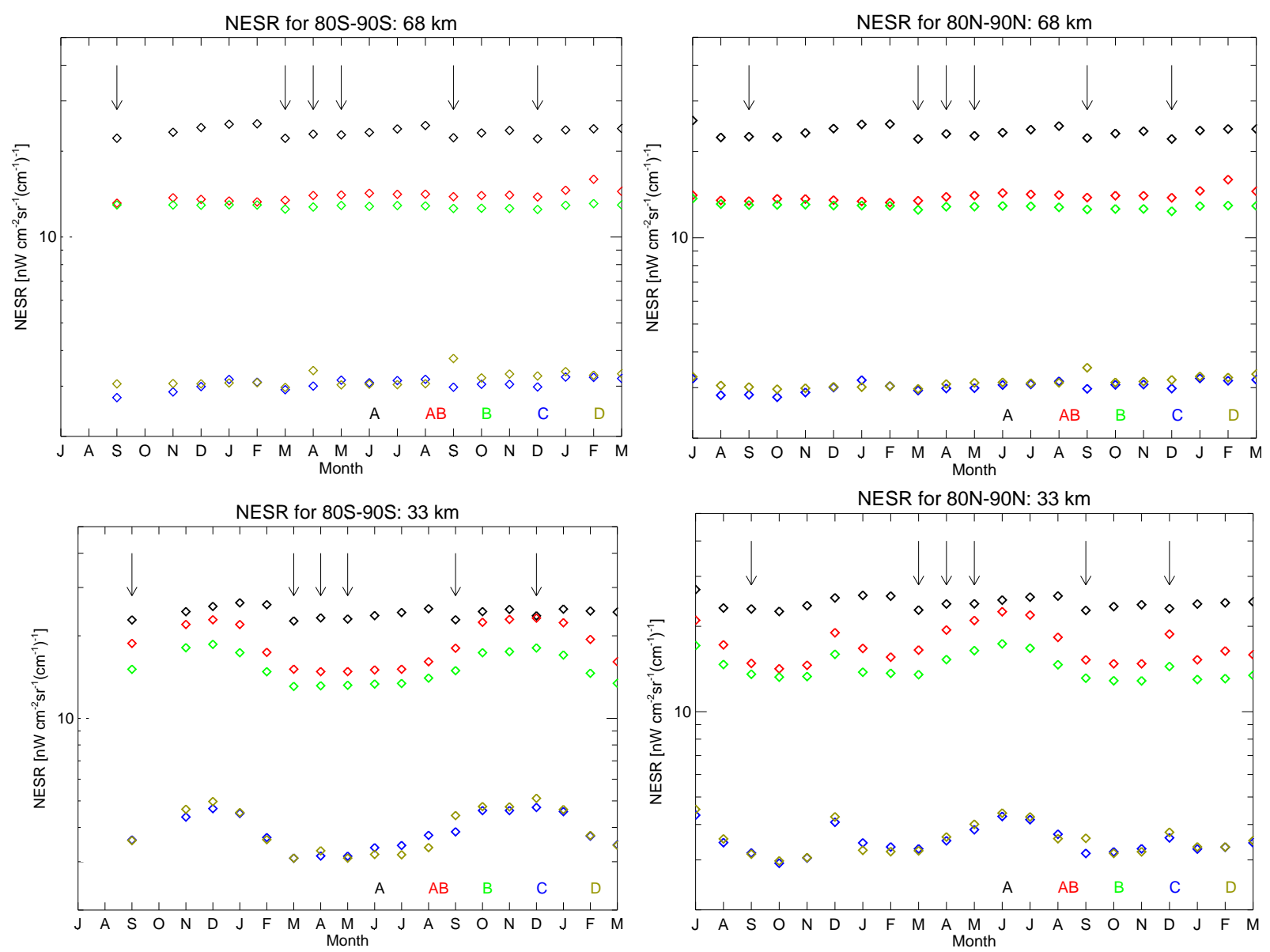

Fig. 4. Time series of NESR at $68 \mathrm{~km}$ and $33 \mathrm{~km}$ tangent altitudes monthly averaged over 5 different spectral bands $\left(\mathrm{A}: 685-970 \mathrm{~cm}^{-1}\right.$, AB: $1020-1170 \mathrm{~cm}^{-1}$, B: $1215-1500 \mathrm{~cm}^{-1}$, C: $1570-1750 \mathrm{~cm}^{-1}$, D: $\left.1820-2410 \mathrm{~cm}^{-1}\right)$. Southern Hemisphere gaps correspond to the missing Antarctic L2 profiles for July-August 2002 and October 2002. Arrows indicate decontamination events. 
allow for any systematic difference between the tangent pressures of the ascending and descending profiles (associated with any relative altitude offset) it was first necessary to adjust descending profile value $x_{2}$ to the same tangent pressure as the ascending profile value $x_{1}$ by applying the following correction:

$x_{2}\left(p_{1}\right)=x_{2}\left(p_{2}\right)+\left(\frac{d x}{d p}\right)\left(p_{1}-p_{2}\right)$

where $p_{1}$ and $p_{2}$ are the tangent pressures at the corresponding levels in profiles 1 and 2 respectively, and $(d x / d p)$ is the average gradient of target species with pressure at that level, determined from the full set of profiles for that latitude/month. The use of the averaged gradient, rather than a simple pressure interpolation on a profile-by-profile basis, is to avoid introducing any extra smoothing (hence a reduction in random error) to profile 2.

The mean $\bar{z}$ and variance $\sigma_{z}^{2}$ of differences $z_{i}$ belonging to the same statistical distribution are then defined in the usual way:

$$
\begin{aligned}
\bar{z} & =\frac{1}{N} \sum_{i=1}^{N} z_{i} \\
\sigma_{z}^{2} & =\frac{1}{N-1} \sum_{i=1}^{N}\left(z_{i}-\bar{z}\right)^{2}
\end{aligned}
$$

where $N$ is the number of matching pairs $(\sim 100 \mathrm{~s})$ at each profile level within each latitude band/month.

If we assume that each profile value $x_{i}$ measures the "true" atmospheric value with the same mean bias and precision $\sigma_{x}$ then, for a large statistical sample,

$$
\begin{aligned}
\sigma_{z}^{2} & =\left\langle\left(\left(x_{1} \pm \sigma_{x}\right)-\left(x_{2} \pm \sigma_{x}\right)\right)^{2}\right\rangle \\
& \simeq 2 \sigma_{x}^{2} .
\end{aligned}
$$

where $\sigma_{x_{1}}=\sigma_{x_{2}}=\sigma_{x}$.

The standard deviation (SD) used in the following is therefore $\sigma_{x} \simeq \sqrt{\sigma_{z}^{2} / 2}$.

However, before making this comparison, there is another contribution to the observed scatter $\sigma_{z}^{2}$ in the concentration profiles that should be considered.

\subsection{Pressure and temperature $(\mathrm{pT})$ error propagation}

In the ESA processing the temperature and tangent pressure are retrieved first, then the VMR profiles.

Errors in the pT retrieval are known to have a significant effect on the subsequent VMR retrievals and, for this study in particular, the propagation of the random errors from the pT retrieval $\left(\mathbf{V}_{p T}\right)$ should be taken into account when considering the random variability of VMR profiles. This can be expressed as an additional error covariance $\mathbf{V}_{x}^{p T}$

$\mathbf{V}_{x}^{p T}=\mathbf{E} \mathbf{V}_{p T} \mathbf{E}^{T}$
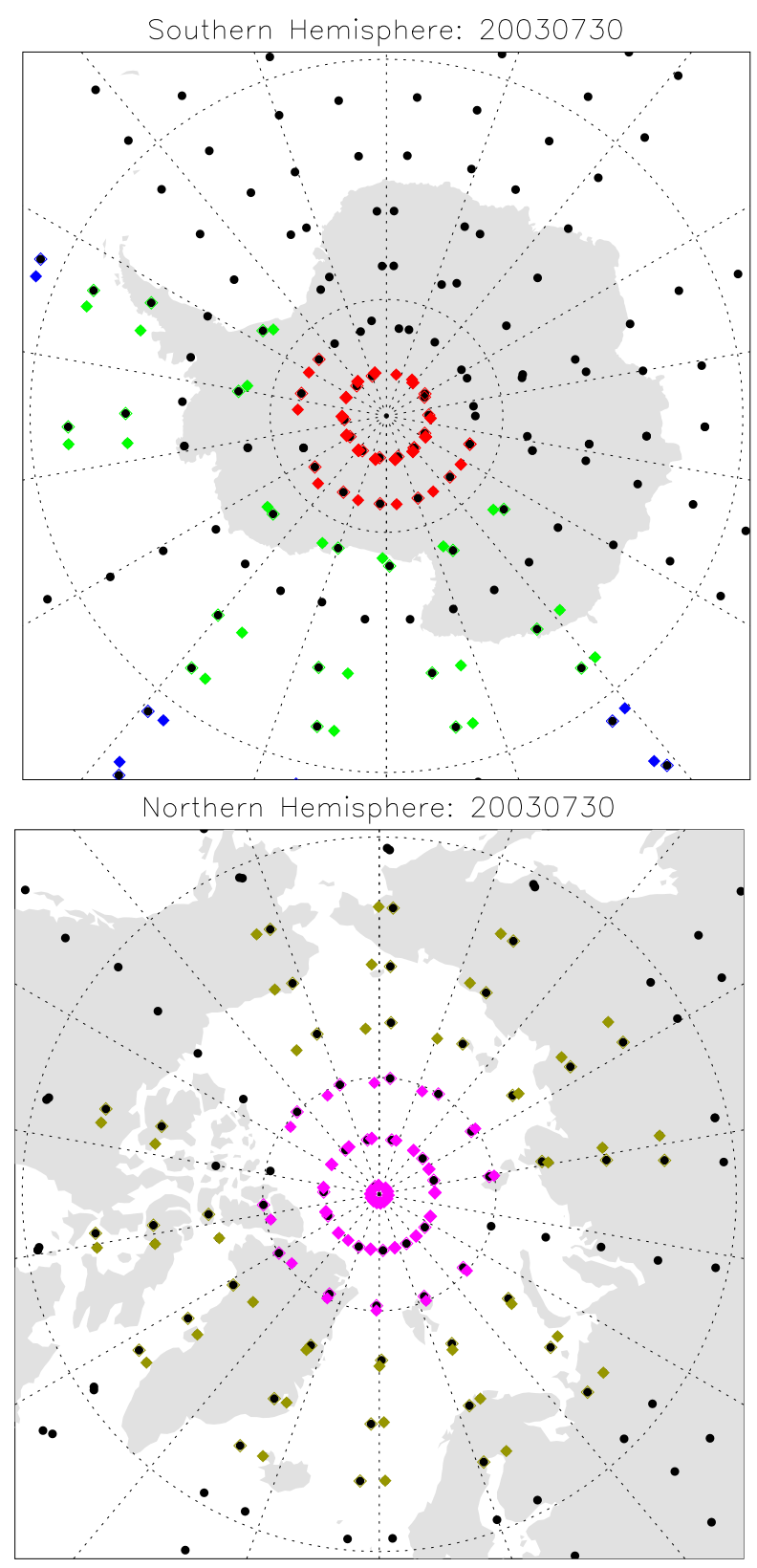

Fig. 5. Locations of the ESA L2 profiles of MIPAS for 30 July 2003. Colored points represent positions where orbital tangent tracks intersect when two observations are made of the same atmosphere $3 \mathrm{~h}$ apart (red for South Pole and magenta for North Pole) and $12 \mathrm{~h}$ apart (green, blue and yellow). The black orbit intersections in the South Pole regions come from observations made more than $12 \mathrm{~h}$ apart.

where $\mathbf{E}$ represents the pT error propagation matrix (Raspollini et al., 2000).

A modified definition of VMR profile precision is then obtained by summing the matrices $\mathbf{V}_{x}^{p T}+\mathbf{V}_{x}$ prior to taking the square roots of the diagonals. 

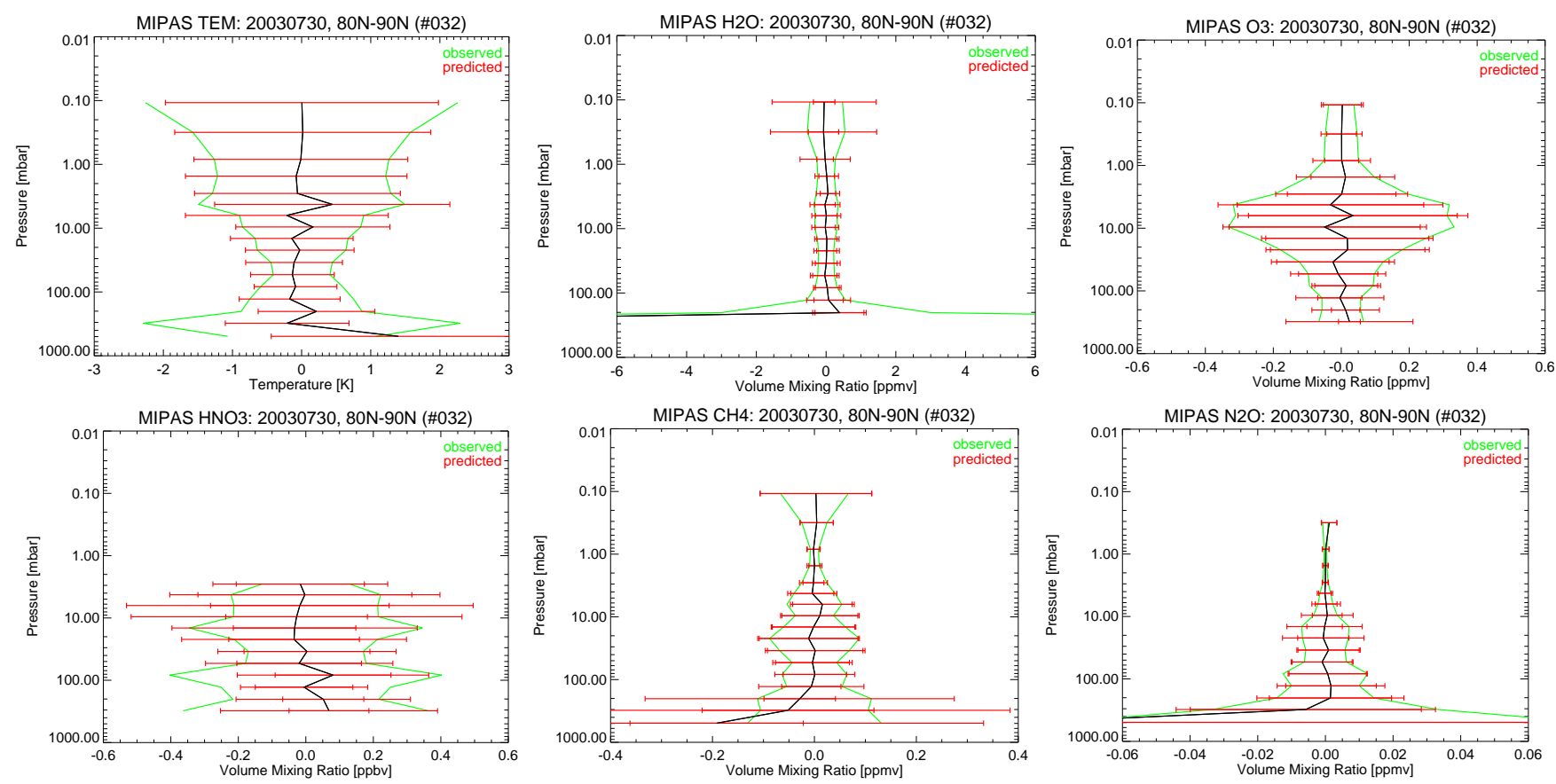

Fig. 6. Example of polar summer comparisons at $80 \mathrm{~N}-90 \mathrm{~N}$ latitudes for 30 July 2003. It shows comparisons for temperature (top left), $\mathrm{H}_{2} \mathrm{O}$ (top middle) $\mathrm{O}_{3}$ (top right), $\mathrm{HNO}_{3}$ (bottom left), $\mathrm{CH}_{4}$ (bottom middle) and $\mathrm{N}_{2} \mathrm{O}$ (bottom right) for 32 pairs of L2 profiles. The black line is the bias between the matched pairs profiles, the red bars are the precision values (for the VMR plots the pT induced error is included and shown as an additional contribution to the red bars) and the green line is the standard deviation of the ensemble of the matching profile pairs.

The covariance matrix $\mathbf{V}_{x}^{p T}$ is included as part of the L2 product but in practice there is some ambiguity over the units and, in any case, the matrix is only calculated for the cases where spectra for all tangent heights within the retrieval range are available (e.g. cloud-free atmospheres).

Instead, here we use the originally-defined $\mathbf{E}$ matrices (Raspollini et al., 2000), $\mathbf{E}=\mathbf{D C}$, where $\mathbf{D}$ is the VMR retrieval inversion matrix, which is assumed locally independent on pressure and temperature, and $\mathbf{C}$ is the matrix accounting for the $\mathrm{pT}$ error propagation in the simulated spectra of VMR retrieval (i.e. the jacobian of the simulated spectra with respect to pT). Then we have applied Eq. (7), which shows that the $\mathrm{pT}$ propagation error contributes up to an additional $10 \%$ random variability, depending on the species.

\section{Results of the validation}

Figure 6 shows an example (Arctic summer of 30 July 2003) of the comparisons between the standard deviation of the ensemble of profile pairs and the precision values given in the MIPAS data for all target species except for $\mathrm{NO}_{2}$ (which has a large diurnal variation between ascending/descending intersections). The $\mathrm{pT}$ error propagation has been included in the precision values for the VMR profiles.
The temperature comparison (top left panel) is in good agreement at most altitudes, although the standard deviation is much larger than the precision below the $100 \mathrm{mb}$ surface.

The VMRs standard deviation is generally consistent with the precision. For $\mathrm{CH}_{4}$ and $\mathrm{O}_{3}$ at low altitudes and in general for $\mathrm{HNO}_{3}$, the $\mathrm{pT}$ induced error has a large contribution to the precision. For $\mathrm{H}_{2} \mathrm{O}$, the standard deviation of the profile pairs is consistent with the precision except for the last altitude $(\sim 6 \mathrm{~km})$.

To examine the time and latitude dependence of the SD compared to precision, the vertically averaged values of the ratio $\mathrm{SD} /$ precision have been calculated for profile pairs from 4 latitude bands for 5 days each month throughout the full resolution dataset. Some filtering out of unreasonable profile values has been applied.

In general the standard deviation of the ensemble of matching profile pairs should be larger than the precision since it is an approximation of the random uncertainties and it includes the variability of the atmosphere. On the other hand, the precision could be underestimated since it is obtained from a linear error propagation analysis while the retrieval process is expected to be non-linear.

Figure 7 shows the results for the "stratospheric" component, defined as the nominal tangent altitudes from $68-15 \mathrm{~km}$ and Fig. 8 the "tropospheric" component, 12-6 km. 

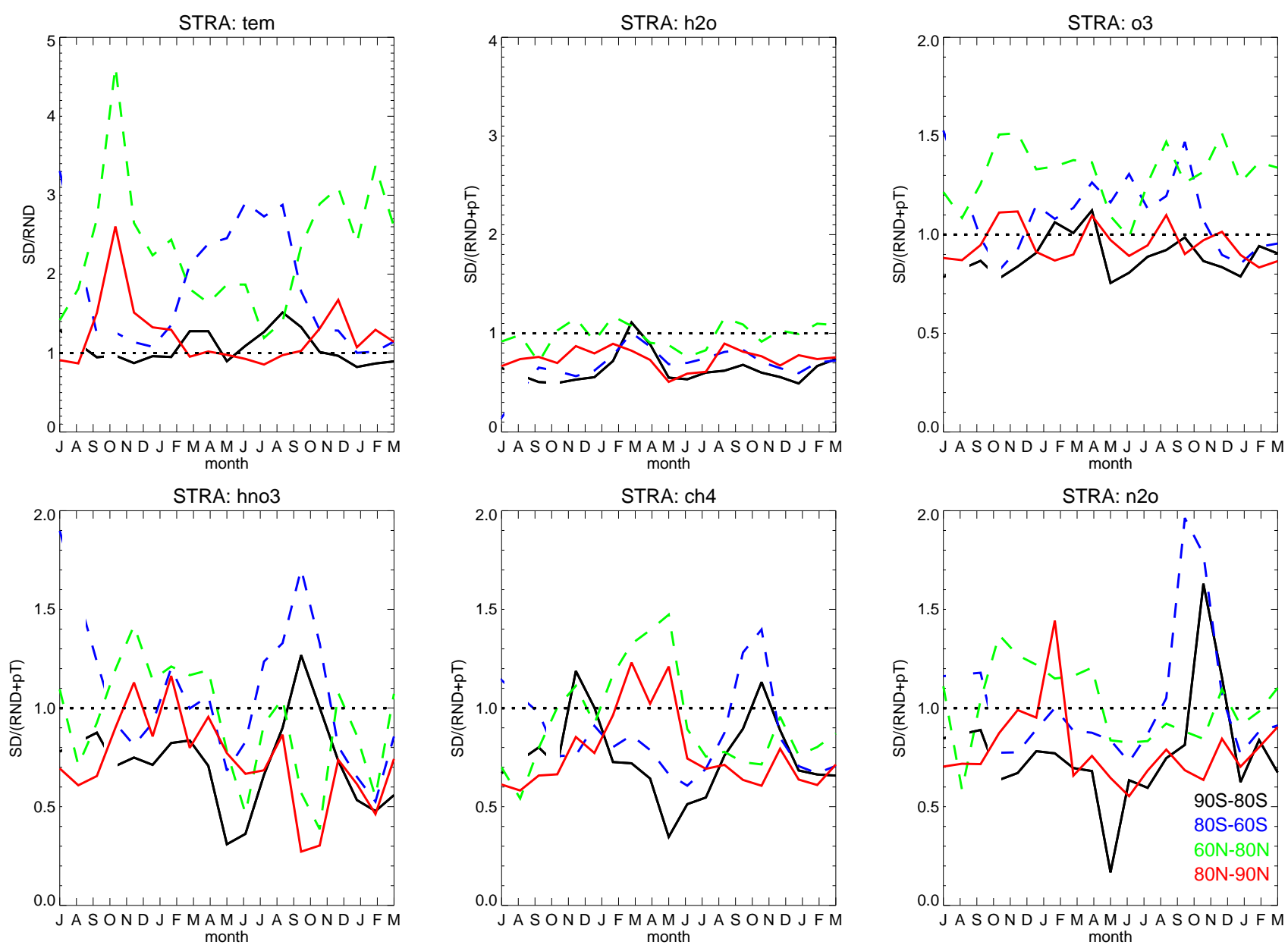

Fig. 7. Time series of the ratio standard deviation/precision for each species derived from the analysis of the matching ascending/descending profile pairs from July 2002 to March 2004. This ratio is computed averaging the whole standard deviation/precision profiles down to the nominal tangent height of $15 \mathrm{~km}$ (i.e. stratosphere). For each species, Black indicates $80 \mathrm{~S}-90 \mathrm{~S}$, Red $80 \mathrm{~N}-90 \mathrm{~N}$, Blue $60 \mathrm{~S}-80 \mathrm{~S}$ and Green $60 \mathrm{~N}-80 \mathrm{~N}$ regions as a function of time. Solid lines indicate matching pairs within $3 \mathrm{~h}$, while dashed lines within $12 \mathrm{~h}$. The precision includes the precision values given in the MIPAS L2 data and the pT error propagation component for the VMR target species plots.

Since there is no significant difference between polar summer and polar winter in the ratio (Fig. 7), whereas the atmospheric variability is significantly larger in polar winter, it seems that the atmospheric variability does not significantly affect the results.

In general, the ratio is closer to the predicted value of 1 for the stratospheric component than the tropospheric component, i.e. the precision appears to more accurately represent the random error in the stratosphere than the troposphere.

For temperature particularly, the ratio appears larger for winter conditions but for other molecules (stratospheric $\mathrm{HNO}_{3}, \mathrm{CH}_{4}$ and $\mathrm{N}_{2} \mathrm{O}$ ) the peak seems to occur in the southern hemisphere around September/October 2003.

Tropospheric ratios for $\mathrm{O}_{3}, \mathrm{CH}_{4}$ and, usually $\mathrm{HNO}_{3}$ are nearer 0.5 than 1 , suggesting that the random error is overestimated by a factor 2 .
There are various sources of pseudo-random error associated with the retrieval beyond those represented by the NESR and pT error propagation. Many of these are associated with features present in the atmospheric spectra which cannot be represented in the forward model. One of these is associated with assuming a horizontally homogeneous atmosphere (particularly in temperature) along the line-of-sight. Measurements at limb average the atmosphere over long horizontal distances and the profile at the tangent points is sheared horizontally. Moreover, the retrieved value at one tangent altitude is used to model the contribution of the atmosphere along the line of sight for lower altitudes, even though these altitudes are at different locations. Another source of pseudo-random error is associated with features with vertical structures sharper than the $3 \mathrm{~km}$ profile level spacing (e.g. the tropopause, residual cloud, tropospheric water vapour 

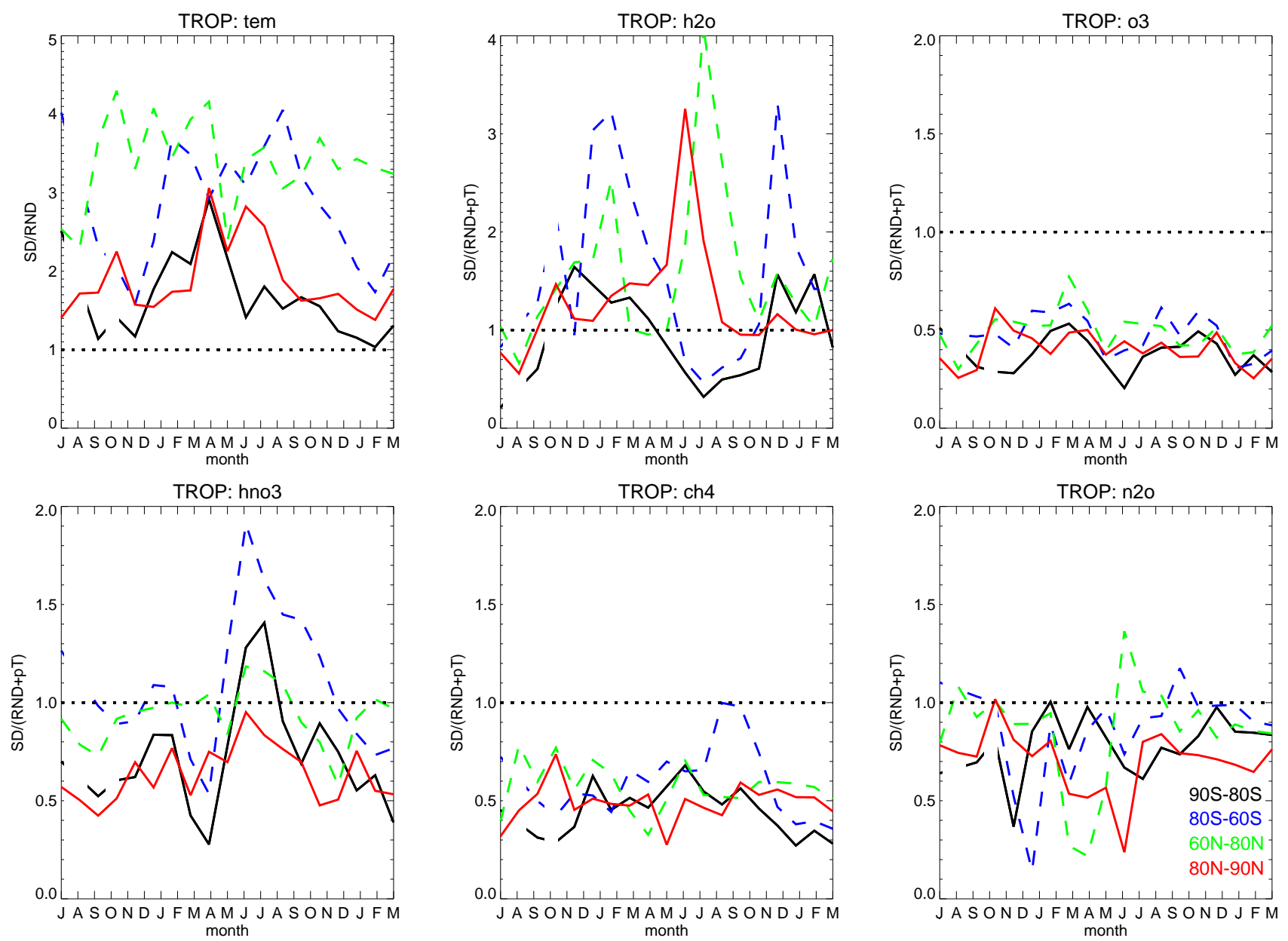

Fig. 8. Same as Fig. 7 where instead of averaging over the whole profile down to $15 \mathrm{~km}$, the average is only over the lowest three nominal tangent heights from 6 to $12 \mathrm{~km}$ (i.e. troposphere).

gradient) which are not resolved by the $3 \mathrm{~km}$ field of view of the instrument. This leads to incorrect modelling of the radiance and the sensitivity of the retrieval to systematic errors within the forward model representation of the atmosphere. The influence of non-detected clouds, due to not sufficiently stringent cloud index threshold to remove all significant cloud contamination, might also be a source of pseudorandom error associated with the retrieval.

These approximations tend to trigger instabilities in the retrieval, causing oscillations in the profile which may be in the opposite phase for two slightly different viewing conditions. In particular the horizontal temperature gradients may explain the larger SD observed in winter conditions and the unresolved vertical structure the increased tropospheric SD for temperature and $\mathrm{H}_{2} \mathrm{O}$.

By contrast, a ratio less than unity implies an overestimate of the random error. This applied most obviously to the two molecules $\left(\mathrm{HNO}_{3}\right.$ and $\left.\mathrm{O}_{3}\right)$ where the tropospheric concen- trations are relatively low compared to the stratosphere. Although not evident from Fig. 6, the relative random error for the troposphere is much larger for these molecules so the underestimate may be associated with the breakdown of the assumption of linearity associated with Eq. (1).

Moreover, in the case of $\mathrm{HNO}_{3}$ a large number of unrealistically small positive numbers (set to $10^{-10}$ in the retrieval instead of negative values) has been excluded from the comparison. Although for $\mathrm{HNO}_{3}$ this is due to fluctuations of the noise rather than retrieval problems, the result is limited by comparing only positive profiles.

\section{Conclusions}

The main source of the random error of the ESA L2 MIPAS profiles is the error due to the mapping of the radiometric noise in the retrieved profiles. This varies mainly with the atmospheric temperature, which controls the total radiance 
received. An additional contribution to the random error for the target species profile is the pressure and temperature propagation error.

The precision varies with time in the polar regions and it shows a distinct increase in the polar winters for all target species, which can be explained by a reduction in radiance received from the colder atmosphere. The noise itself varies with time, steadily rising between ice decontamination events, but the Level 2 precision has a greater variation due to the atmospheric temperature. Hence, the precision varies latitudinally/seasonally with temperature, with a small superimposed temporal structure determined by the degree of ice contamination on the detectors.

By comparing MIPAS retrievals at orbit intersections (effectively comparing two observations of the same atmosphere) a check can be made of the actual scatter in the measurements. For 5 days per month of full resolution MIPAS operation, the standard deviation of the matching profile pairs is computed and compared with the precision given in the MIPAS Level 2 data, except for $\mathrm{NO}_{2}$ since it has a large diurnal variation between ascending/descending intersections.

In general, the standard deviation of the matching pairs is usually a factor 1-2 larger than the precision. The precision appears to more accurately represent the random error in the stratosphere than the troposphere. Larger standard deviations of the matching pairs observed in winter conditions are thought to be due to effects such as horizontal inhomogeneity of the atmosphere along the line-of-sight, while unresolved vertical structure may explain the increased tropospheric standard deviations for temperature and $\mathrm{H}_{2} \mathrm{O}$.
Acknowledgements. The authors would like to thank J. Hurley for the efficient help on the provision of MIPAS L1B data. The MIPAS experiment is supported by the European Space Agency (ESA). This work is supported by DARC (Data Assimilation Research Centre), UK.

Edited by: P. Hartogh

\section{References}

ESA, SP-1229: ESA ENVISAT-MIPAS: an instrument for atmospheric chemistry and climate research, European Space Agency, ESTEC, Noordwijck, The Netherlands, 2000.

Kleinert, A., Aubertin, G., Perron, G., et al.: MIPAS Level 1B algorithms overview: operational processing and characterization, Atmos. Chem. Phys., 7, 1395-1406, 2007, http://www.atmos-chem-phys.net/7/1395/2007/.

Lambert, A., Grainger, R. G., Remedios, J. J., et al.: Validation of aerosol measurements from the improved stratospheric and mesospheric sounder, J. Geophys. Res., 10(D6), 9811-9830, 1996.

Raspollini, P. and Ridolfi, M.: Mapping of temperature and line-ofsight errors in constituent retrievals for MIPAS/ENVISAT measurements, Atmos. Environ., 34, 5329-5336, 2000.

Raspollini, P., Belotti, C., Burgess, A., et al.: MIPAS level 2 operational analysis, Atmos. Chem. Phys., 6, 5605-5630, 2006, http://www.atmos-chem-phys.net/6/5605/2006/.

Ridolfi, M., Carli, B., Carlotti, M., et al.: Optimised forward model and retrieval scheme for MIPAS near-real-time data processing, Appl. Opt., 39(8), 1323-1340, 2000. 